\title{
Intra-cycle power: is the flow profile a neglected component of lung protection?
}

\author{
John J. Marini ${ }^{*}{ }^{*}$, Philip S. Crooke ${ }^{2}$ and Luciano Gattinoni ${ }^{3}$
}

(0) 2021 Springer-Verlag GmbH Germany, part of Springer Nature

An uncontested clinical objective of mechanical ventilation is to avoid further injury to lung tissue (VILI). Welldeserved clinical attention has been given to constraining tidal volume, and the end-inspiratory ('plateau') and driving pressures $[1,2]$. Yet, because damaging tidal strains require repeated energy expenditure, awareness has been raised regarding the importance of cycling frequency that determines the energy imparted per minute [3]. At the bedside, tidal energy per passive inflation cycle is the product of delivered volume (the integral of flow) and the inflation pressure above the relaxed baseline needed to deliver it. In a simplified model, the total airway pressure component of energy is the sum of flow-resistive pressure, driving pressure, and the initial pressure (PEEP).

In current clinical parlance, cumulated inflation energy per minute has been termed mechanical 'power', the product of inflation work per cycle and ventilating frequency. However, not all combinations of pressure, volume and frequency that sum to the same power value by this definition generate enough strain per cycle to advance parenchymal damage. The amplitudes of plateau and driving pressures as well as the frequency of their repetition are keys to safety or danger $[4,5]$.

We believe that flow magnitude and flow profile of the individual cycle (e.g., constant or decelerating) are relatively neglected among the adjustable ventilator settings that are relevant to lung protection. If so, why should these flow characteristics matter clinically?

Flow rate not only determines the velocity of flow through the airways, but also the expansion rate of

\footnotetext{
*Correspondence: marin002@umn.edu

1 Pulmonary and Critical Care Medicine, University of Minnesota and Regions Hospital, MS 11203B, 640 Jackson St., Saint Paul, MN 55101, USA

Full author information is available at the end of the article
}

parenchymal units. Viewed at the micro level, flow-determined rates of alveolar expansion may amplify or attenuate the strains experienced by individual units, due to local geometry and interdependence, inherent mechanical properties, and surrounding viscoelastic microenvironments, which include the vascular side [6]. Note that the VILI-relevant expansion velocity is not externally measured flow but flow relative to the diminished number of open lung units within the 'baby lung' [7].

Power can be evaluated on any time scale. Thus, while the 'power' defined as the product of per-cycle energy and frequency is certainly of clinical interest for lung protection [3], essentially, it is a cumulative measure of the rate of energy delivery and as such, an incomplete tracker of VILI risk. In fact, injury hazard is co-determined by tissue micro-strains experienced during expansion. Discounting local stress and strain amplifiers, the product of externally measured flow and the developed pressure quantifies the 'intra-cycle' power for the entire lung. These measurable moment-by-moment energy loads can be estimated by specifying the flow profile, the targeted tidal volume, the elapsed time from inflation onset, PEEP and the pressure components of inflation determined by compliance and resistance. However the VT is delivered, the total inflation energy at the alveolar level is virtually the same at end-inflation. Nonetheless, the flow profiles determine how that energy distributes as the lung inflates, influencing the instantaneous strains within the expanding lung.

The flow-resistive energy component of airway pressure dissipates in moving gas across the endotracheal tube and through the native airways, and in displacing parenchymal tissue to higher volumes. At end-inflation the 'elastic' component that is built and conserved during inflation is comprised of two subcomponents-driving pressure and PEEP. Intracycle power building to these elastic pressures can be thought of as the instantaneous

\section{Springer}


a

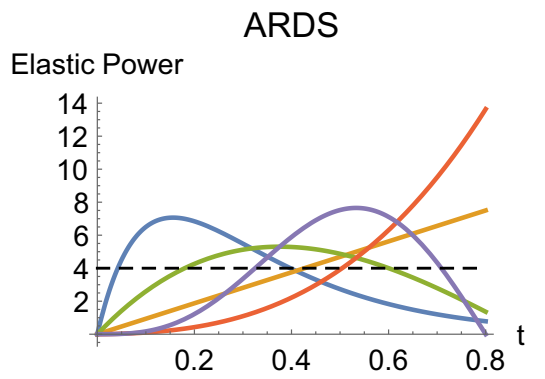

C

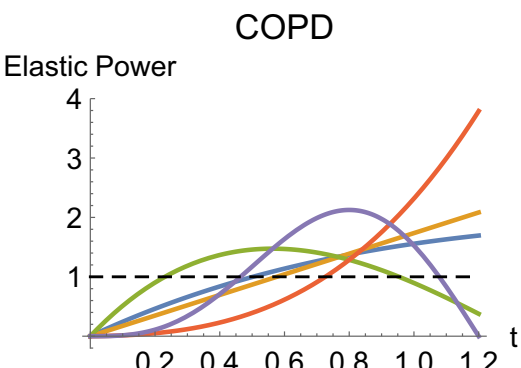

— Pset

CF

- DF

- $\mathrm{AF}$

$\longrightarrow$ Sin

- Pset

CF

$\longrightarrow$ DF

- AF

$-\operatorname{Sin}$ b

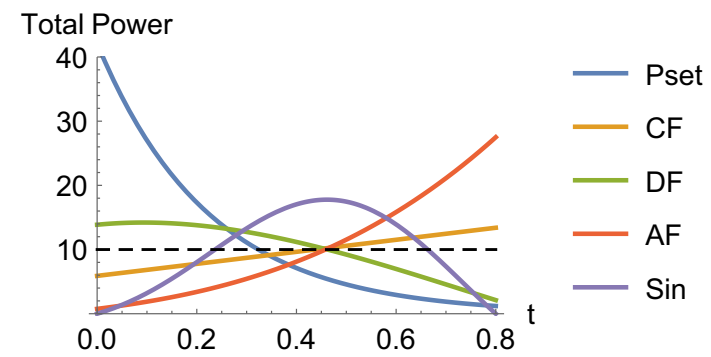

d

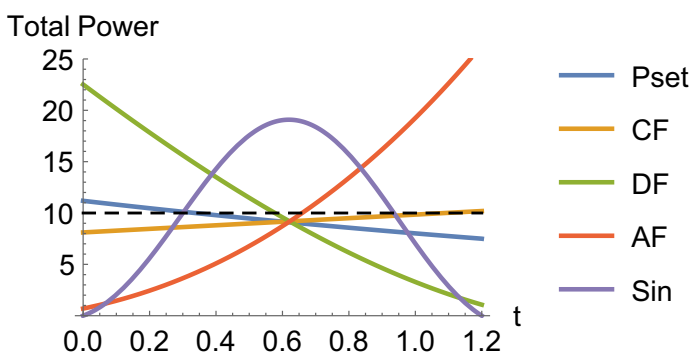

Fig. 1 Hypothetical intracycle elastic ('alveolar') and total power curves as mathematically modeled functions of the elapsed inspiratory time (t) during a single inflation to the same tidal volume for 5 flow profiles: constant (CF), decelerating (DF), sinusoidal (Sin), accelerating (AF) pressure control (Pset). These modes are compared for typical clinical values simulating severe ARDS $(\mathbf{a}, \mathbf{b})$ and severe COPD (c, $\mathbf{d})$, and a hypothetical threshold level (dashed line) demarcates the lower boundary of VILI risk. Note that for each patient type, marked numerical differences separate instantaneous total and elastic power values, as well as the flow-driven power profiles (Zero PEEP is assumed)

inflation energy being applied at the alveolar level ('elastic power'), whereas power that includes resistive pressure losses is 'total' power applied to the entire respiratory system. In this evolving field of VILI energetics, it is not yet clear whether elastic power (the component most relevant to alveolar energy load), or total power (which includes energy lost through the large and small airways), is most relevant to VILI $[4,8]$.

\section{Threshold for damaging strain}

Conceptually, thresholds of pressure and power demarcate entry into the zone of heightened injury hazard. Intracycle power applied in excess of the threshold risks inflicting damage by overstraining matrix microelements that bind individual alveolar units [9]. Again in theory, such thresholds would vary with the lung's vulnerability to strain and the local anatomic position of the lung unit in question. For example, a normal-sized healthy lung should begin to mechanically injure at a high-pressure threshold, whereas the non-homogeneous baby lung of acute respiratory distress syndrome (ARDS) would yield to a much lower one. Dependent lung units would have different (presumably lower) thresholds than ones situated non-dependently. The liklihood and timing of threshold crossing as well as the impact of above-threshold power are strongly influenced by the flow pattern used: constant (CF), decelerating (DF), sinusoidal (Sin), and accelerating (AF). Pressure control (Pset), although targeted to airway pressure, provides a decelerating flow pattern. The intracycle total and elastic power profiles that these flow profiles generate vary considerably with mechanical properties (resistance and compliance) and ventilator settings (Fig. 1). Interestingly, the sinusoidal flows of natural spontaneous breathing are seldom employed during passive inflation.

\section{Potential clinical implications of this hypothesis}

At the bedside, flow magnitude and profile, the keys to intracycle power delivery, receive ample attention as determinants of patient-ventilator dys-synchrony [10], but, as yet, these have been given scant consideration as contributors to VILI risk. Nonetheless, experimental evidence suggests that high flow 'spikes' (e.g., pressure control) have greater damaging potential than those without [11]. Intriguingly, the wisdom of limiting peak flow may apply to the earliest phase of expiration, as well [12].

Assuming the importance of flow pattern translates clinically, what are the immediate implications for lung protection? In our view, they are: (1) to reduce minute ventilation requirements that drive frequency and 
magnitude of inspiratory flow, e.g., by permissive hypercapnia, extracorporeal $\mathrm{CO}_{2}$ removal; (2) to select a flow profile and I:E ratio that minimizes high flow and power spikes; (3) to take measures that reduce amplifiers of flow-determined power, e.g., lower tidal volumes and recruitment by judicious PEEP to increase the capacity of the baby lung to accept tidal flow without overdistention, and prone positioning to minimize mechanical nonhomogeneity. Clinicians already focus on tidal pressures and volumes; should a smart strategy for lung protection also pay close attention to flow requirements, flow profile and intra-cycle power?

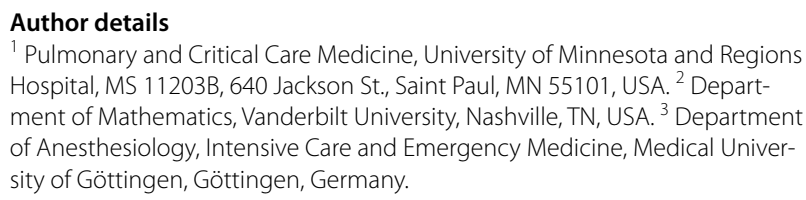

1 Pulmonary and Critical Care Medicine, University of Minnesota and Regions Hospital, MS 11203B, 640 Jackson St., Saint Paul, MN 55101, USA. ${ }^{2}$ Department of Mathematics, Vanderbilt University, Nashville, TN, USA. ${ }^{3}$ Department of Anesthesiology, Intensive Care and Emergency Medicine, Medical University of Göttingen, Göttingen, Germany.

\section{Conflicts of interest}

The authors affirm that none has a conflict of financial or other interest relevant to this work.

\section{Publisher's Note}

Springer Nature remains neutral with regard to jurisdictional claims in published maps and institutional affiliations.

Received: 8 February 2021 Accepted: 21 February 2021

Published online: 2 April 2021

\section{References}

1. Slutsky AS, Ranieri VM (2013) Ventilator-induced lung injury. N Engl J Med 369:2126-2136. https://doi.org/10.1056/NEJMra1208707
2. Thompson BT, Chambers RC, Liu KD (2017) Acute respiratory distress syndrome. N Engl J Med 377:562-572. https://doi.org/10.1056/NEJMr a160807

3. Gattinoni L, Tonetti T, Cressoni M, Cadringher P, Herrmann P, Moerer O, Protti A, Gotti M, Chiurazzi C, Carlesso E, Chiumello D, Quintel M (2016) Ventilator related causes of lung injury: the mechanical power. Intensive Care Med 42:1567-1575. https://doi.org/10.1007/s00134-016-4505-2

4. Marini JJ, Rocco PR, Gattinoni L (2020) Static and dynamic contributors to ventilator-induced lung injury in clinical practice pressure, energy, and power. Am J Respir Crit Care Med 201 (7):767-777

5. Amato MB, Meade MO, Slutsky AS, Brochard L, Costa EL, Schoenfeld DA et al (2015) Driving pressure and survival in the acute respiratory distress syndrome. N Engl J Med 372:747-755

6. Faffe DS, Zin WA (2009) Lung parenchymal mechanics in health and disease. Physiol Rev 89:759-775

7. Marini JJ, Gattinoni L (2020) Time course of evolving ventilator-induced lung injury: the "shrinking baby lung." Crit Care Med 48:1203-1209

8. Marini JJ (2020) Rocco PRM Which component of mechanical power is most important in causing VILI? Crit Care 24:39. https://doi.org/10.1186/ s13054-020-2747-4

9. Hynes RO (2009) The extracellular matrix: not just pretty fibrils. Science 326:1216-1219

10. Maeda Y, Fujino Y, Uchiyama A, Matsuura N, Mashimo T, Nishimura M (2004) Effects of peak inspiratory flow on development of ventilatorinduced lung injury in rabbits. Anesthesiology 101:722-728

11. Subira C, de Haro C, Magrans R, Fernandez R, Blanch L (2018) Minimizing asynchronies in mechanical ventilation: current and future trends. Respir Care 63(4):464-478

12. Schmidt J, Wenzel C, Spassov S, Borgmann S, Lin Z, Wollborn J, Weber J, Haberstroh J, Meckel S, Eiden S, Wirth S, Schumann S (2020) Flowcontrolled ventilation attenuates lung Injury in a porcine model of acute respiratory distress syndrome: a preclinical randomized controlled study. Crit Care Med 48(3):e241-e248. https://doi.org/10.1097/CCM.0000000000 004209 